\title{
Expanding capabilities and infrastructure for cryo-EM
}

\author{
Many challenges and considerations must be evaluated when expanding and supporting new cryo-electron \\ microscopy facilities.
}

\author{
Kutti R. Vinothkumar
}

T: he importance of structural biology research has been reinforced during the ongoing COVID-19 pandemic, as newly determined viral protein structures have helped us understand the mode of action of antibodies and drugs against SARS-CoV-2 and its variants. This success is built on decades of work toward the development of various structural biology techniques.

Among these, cryo-electron microscopy (cryo-EM) has recently seen rapid progress, with structures of many previously intractable macromolecules now being determined. This is largely because it requires very little protein and can image proteins as individual molecules that can be averaged to produce high-resolution three-dimensional atomic models. In fact, structures have recently been obtained without a need for extensive purification from lysates ${ }^{1}$, and the power of seeing and identifying protein molecule from the images (or class averages) is an added asset (Fig. 1). With better sample preparation methods, next-generation detectors and new image processing software, it will be possible to obtain even better images and structures ${ }^{2}$. Single-particle cryo-EM has become and will continue to grow as a routine method, and in the next few years the time taken from gene to structure is bound to shorten.

A natural extension of imaging with electron microscopy is in situ structure determination. Large molecules at very high resolution can already be studied in the cellular environment ${ }^{3}$, and this is likely to become even more powerful. The future challenge lies in imaging low-copy-number macromolecules and membrane protein complexes in their native environment. These proteins are often heterogenous in their composition. Integration of different techniques, including light and electron microscopy, mass spectrometry and genetics, will help us realize this goal.

Synchrotron-based models have been very successful across the world, and installation of cryo-EM (and other) facilities within them has been a wise move. Not

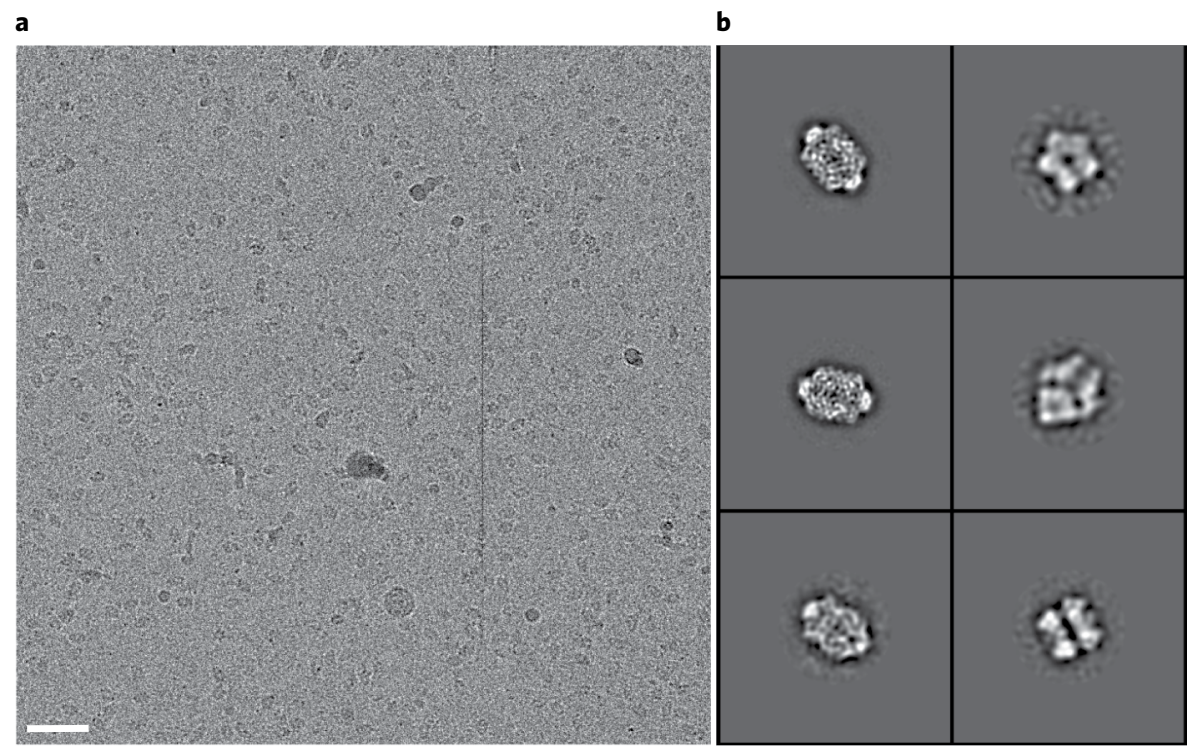

Fig. 1 | Cryo-EM (or EM in general) can help improve the biochemical quality of the protein for both compositional and conformational heterogeneity. a, A demonstration of the power of cryo-EM (scale bar, $500 \AA$ ). The micrograph reveals a number of different particles; to a trained eye, some of these molecules might be familiar. $\mathbf{b}$, After two-dimensional classification, the identity of the molecule may become clear (box size, $414 \AA$ ), and subsequent mass spectrometry can confirm the identification. Image courtesy Shaileshanand Jha, a graduate student at NCBS, Bangalore. Cryo-EM can produce structures of macromolecules from a mixture without purification of a single entity? Whether such an approach will become the norm remains to be seen.

many countries have such integrated facilities, and structural biology in India has been an uphill task, in particular when it comes to access to brighter synchrotron sources ${ }^{4}$. When working on competitive projects, easy access to a synchrotron facility (preferably at a local site) can make a huge difference for rapid scientific progress.

The advent of cryo-EM is great for countries like India as, in contrast to synchrotron facilities, large infrastructure is not needed. India has a few institutes with functional cryo-EM ${ }^{4}$, and several others are planned to come online within the end of the year. This is extremely encouraging. There are also plans in place for training service engineers and application scientists to augment these facilities. In practical terms, it is often easy to obtain the initial funding for the procurement of the instrument and setting up the facilities. However, subsequent long-term support to cover the costs of maintaining the instrument and providing easy accessibility to researchers without steep charges will ultimately determine the success of such facilities. The inevitable lockdown due to the pandemic has also highlighted our dependence on research consumables delivered from other countries. For instance, an interruption in supply has meant that we have samples and EM time but not enough EM grids. Being able to source consumables locally could alleviate this dependence. Thus, one of the challenges for us in the near future is to build integrated facilities with good user support and long-term sustainability in order to tackle 
interesting and difficult biological problems across scales.

\author{
Kutti R. Vinothkumar (iD $ه$ \\ National Centre for Biological Sciences TIFR, \\ Bangalore, India. \\ $\bigotimes_{e-m a i l: v k u m a r @ n c b s . r e s . i n}$
}

Published online: 7 May 2021

https://doi.org/10.1038/s41592-021-01139-x

\section{References}

1. Su, C. C. et al. Nat. Methods 18, 69-75 (2021).

2. Naydenova, K., Jia, P. \& Russo, C. J. Science 370, 223-226 (2020).

3. O'Reilly, F. J. et al. Science 369, 554-557 (2020).

4. Subramanian, R., Mayor, S. \& Vinothkumar, K. R. Biophys. Rev. 11, 513-514 (2019)
Acknowledgements

The author acknowledges funding from Department of Biotechnology, India, B-Life grant DBT/PR12422/ MED/31/287/2014; the Department of Atomic Energy, Government of India, under Project Identification No. RTI4006; and SERB, India, for the Ramanujan Fellowship (RJN-094/2017).

Competing interests

The author declares no competing interests.

\title{
RNA structure: a renaissance begins?
}

Check for updates

\section{Advances in cryo-EM technology will open a new era of RNA-only 3D structure determination.}

\author{
Rhiju Das
}

W here are all the RNA structures? For decades, RNA has been known to act as both a genetic script and a molecular machine, and the amount of RNA sequence transcribed in organisms like humans exceeds the number of protein sequences by at least tenfold ${ }^{1}$. Numerous RNA structures have been deposited in the Protein Data Bank (PDB), which, despite its name, remains the biology community's storehouse of structural information for both RNA and proteins. But a quick search in the PDB reveals a serious imbalance tipped in favor of protein structures: the fraction of RNA-only structures remains below $1 \%$. Biologists-even RNA structural biologists-would be forgiven for assuming that most RNAs don't fold. Or, if RNAs do fold, their structures might be ill-defined in three dimensions unless organized by partners like proteins. As a molecule, RNA has been called 'floppy', 'flexible' and 'conformationally heterogeneous', and this reputation has scared off all but the boldest from attempting crystallography or NMR on RNA-only structures. Is determining RNA three-dimensional (3D) structure a lost cause?

A couple of years ago, the field began to revisit the determination of RNA-only structures with cryogenic electron microscopy (cryo-EM), not expecting much but excited by the technique's clear power in imaging ribosomes, spliceosomes and other RNAs complexed to proteins. To everyone's surprise, protein-free, RNA-only complexes in fact did lend themselves to imaging by cryo-EM. Newly visualizable molecules included the Tetrahymena ribozyme, the first RNA-only enzyme discovered in nature (Fig. 1a) ${ }^{2}$; its global structure had remained intractable for four decades. Other RNAs studied using cryo-EM were smaller than $30 \mathrm{kDa}$, well below the $\sim 100$ $\mathrm{kDa}$ detection limit expected for proteins ${ }^{3}$, and maps with sub-4-Å resolution even allowed detection of small-molecule ligands ${ }^{4}$. In 2020, the COVID-19 pandemic prompted

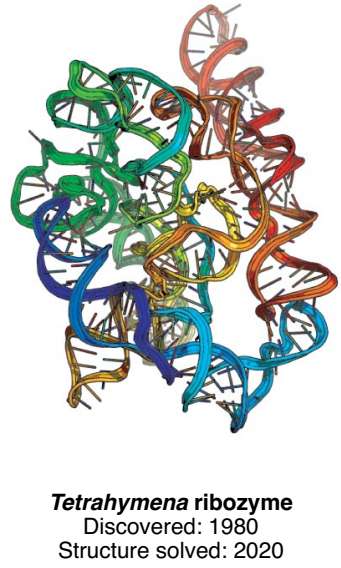

b

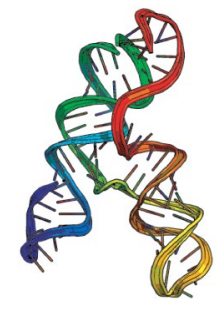

SARS-CoV-2 frameshift element Discovered: 2020 Structure solved: 2020

Fig. 1 | Coordinates of RNA molecules with complex folds revealed through cryogenic electron microscopy. a, Tetrahymena ribozyme, first RNA-only enzyme discovered (PDB 6WLS). b, SARS-CoV-2 frameshift stimulation element, a highly conserved potential target for COVID-19 antivirals that reprograms the ribosome (PDB 6XRZ).

testing of even smaller RNAs, including a 28 $\mathrm{kDa}$ frameshift stimulation element from the SARS-CoV-2 genome-and cryo-EM delivered again (Fig. 1b) ${ }^{5}$. Each of these structures revealed homologies to other RNAs; some structures revealed holes and pockets that might be targeted by drugs for potential biomedical disruption. For RNAs, as for proteins, structure truly can illuminate function.

These results suggest that RNA 3D structures are there for the finding. Indeed, RNA may be the perfect molecule for cryo-EM, and cryo-EM may be the perfect technique for RNA. At the time of writing, however, it is still early days for RNA-only cryo-EM. How far can the resolution of cryo-EM be pushed for RNA-only systems, especially if they form multiple biologically important states? How many RNA domains with well-defined, biologically relevant $3 \mathrm{D}$ structures are there really in the human transcriptome-or the Earth's transcriptome?
If the answers are favorable, an RNA structure renaissance has begun, and in another 50 years our 3D structural database may very well be dominated by RNAs, not proteins. Perhaps the Protein Data Bank will need another name. $\square$

\section{Rhiju Das (1D)}

Departments of Biochemistry and Physics, Stanford University, Stanford, CA, USA.

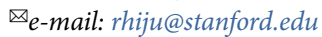

Published online: 7 May 2021

https://doi.org/10.1038/s41592-021-01132-4

References

1. Mattick, J. Nat. Rev. Genet. 5, 316-323 (2004).

2. Kappel, K. et al. Nat. Methods 17, 699-707 (2020).

3. Henderson, R. Q. Rev. Biophys. 28, 171-193 (1995).

4. Zhang, K. et al. Nat. Commun. 10, 5511 (2019).

5. Zhang K. et al. Preprint at bioRxiv https://doi. org/10.1101/2020.07.18.209270 (2020).

The author declares no competing interests. 